\title{
Parallel Algorithm for Detonation Wave Simulation
}

\author{
P. Ravindran and F. K. Lu \\ Aerodynamics Research Center, Mechanical and Aerospace Engineering Department, The \\ University of Texas at Arlington, Arlington, Texas 76019, USA
}

\begin{abstract}
Summary. A parallel algorithm which has sufficient scalability for time accurate solution of a propagating detonation wavefront is developed. The emphasis is placed on reduction of computational time without compromising accuracy. The flow was assumed to be unsteady, inviscid and non-heat-conducting and coupled with finite-rate chemistry. A finite volume formulation was employed to ensure conservation and proper handling of discontinuities. A Runge-Kutta second order scheme was utilized for a time accurate solution, with Roe second order flux splitting scheme for spatial solution. For additional higher order spatial accuracy, van Leer MUSCL technique was employed. Equation stiffness was overcome by point implicit treatment of the source terms. The parallel algortihm was developed using Message Passing Interface standard.
\end{abstract}

\section{Introduction}

Detonation phenomena have been systematically examined theoretically, experimentally and computationally for over a century, beginning with a one-dimensional propagating detonation front as a discontinuity according to the Chapman-Jouguet (CJ) theory $[1,2]$ and the theory of Zel'dovich, Von Neumann and Döring, which has come to be known collectively as the ZND detonation model $[3,4,5]$. The development of numerical techniques for solving high temperature, non-equilibrium flow was pivotal in leading the effort in detonation wavefront simulation. An accurate simulation of the detonation wavefront is a great challenge on computational resources, forcing the use of simplistic approaches which compromises the accuracy of the solution. To overcome these limitations, the solution was initially to employ supercomputers, which in the long run had many inherent difficulties such as their monolithic nature, cooling, bandwidth/latency problems and limited data transmission [6]. In recent years, with the advent of high-performance cluster computing, most of the challenges associated with monolithic supercomputers have been met and addressed.

The development of a parallel algorithm for time accurate solution of a detonation wavefront is reported [7]. The parallel algorithm was developed using the Message Passing Interface standard developed by the Argonne National Laboratory [8] for the purposes of solving the governing equations in a distributed environment.

\section{Governing Equations}

The time-dependent conservation equations are those for an inviscid, non-heat-conducting, reacting gas flow in which thermal nonequilibrium is modeled with a two-temperature approximation. The conservation equations for the individual species are derived first, and then these are combined to yield the complete set of equations. For brevity the 
procedure is summarized here: details can be found in [7]. A simplification comes from the assumption that molecular diffusion is not significant and can be safely neglected when the chemical reactions and the corresponding energy release occur rapidly as in a detonation process. A general representation of species internal energy includes a portion of the internal energy in thermodynamic equilibrium and the remaining portion in a nonequilibrium state. The equilibrium portion of the internal energy is the contribution due to translational and internal modes that can be assumed to be in equilibrium at the translational temperature $T$. The remaining nonequilibrium portion is the contribution due to internal modes that are not in equilibrium at the translational temperature $T$, but may be assumed to satisfy a Boltzmann distribution at a different temperature. For the temperature range of interest in the present study, the rotational mode is assumed to be fully excited and in equilibrium with translational temperature $T$, while the electronic excitation and free electrons modes can be safely ignored.

High temperature flows typically involve some chemical reactions, and the time scale in which chemical reactions take place is important in the estimation of flow field properties. For accurate modeling of a detonation wave, especially in the detonation front where rapid chemical reactions take place in the shock compressed region, species continuity equations based on the chemical kinetics should be solved together with fluid dynamic equations to account for possible chemical nonequilibrium. According to Landau-Teller formulation [10], it is reasonable to assume that the vibrational level of a molecule can change only by one quantum level at a time. This approximation not only reduces the number of species dependent parameters but also simplifies the evaluation of the vibrational relaxation term multiplied by the difference in the translational and vibrational temperature. So, when a point implicit formulation is used on the source terms in the numerical algorithm, the above approximation greatly simplifies an implicit treatment of the temperature difference which drives the relaxation process.

\section{Numerical and Parallel Algorithm Formulation}

A discretized set of equations has been derived from the governing equations using finite volume approach due to its conservative property. Nonequilibrium flows involving finterate chemistry and thermal energy relaxation can be difficult to solve due to stiffness issues associated with varying orders of characteristic time-scales. To overcome this, the point implicit formulation evaluating the source terms at time level $n+1$ has been an effective method used to numerically integrate stiff systems by effectively rescaling all characteristic times in the flow fields into the same order of the magnitude [11]. An extension of Roe scheme to a thermo-chemical nonequilibrium gas has been used for second order accuracy [12] and higher order approximation using MUSCL approach for added spatial accuracy, has been adapted for this work. A modified Newton-Raphson scheme has been used to calculate temperature at each iteration level. For temporal accuracy, a two-step Runge-Kutta scheme has been implemented.

To adapt the numerical solution to a computer cluster, a parallel algorithm has been designed with the primary objectives being scalability and portability. MPI (Message Passing Interface) has been selected due to its inherent advantages of portability acrss different machine architectures, smooth operation in heterogeneous systems, and to allow efficient implementations across machines of differing characteristics [8]. Figure 1 gives a schematic representation of an instance of data transfer in each iteration. The algorithm implemented has an advantage wherein all the values needed from a neighbor are brought 
in one message. Coalescing of communications in this manner reduces the number of messages and improves performance.

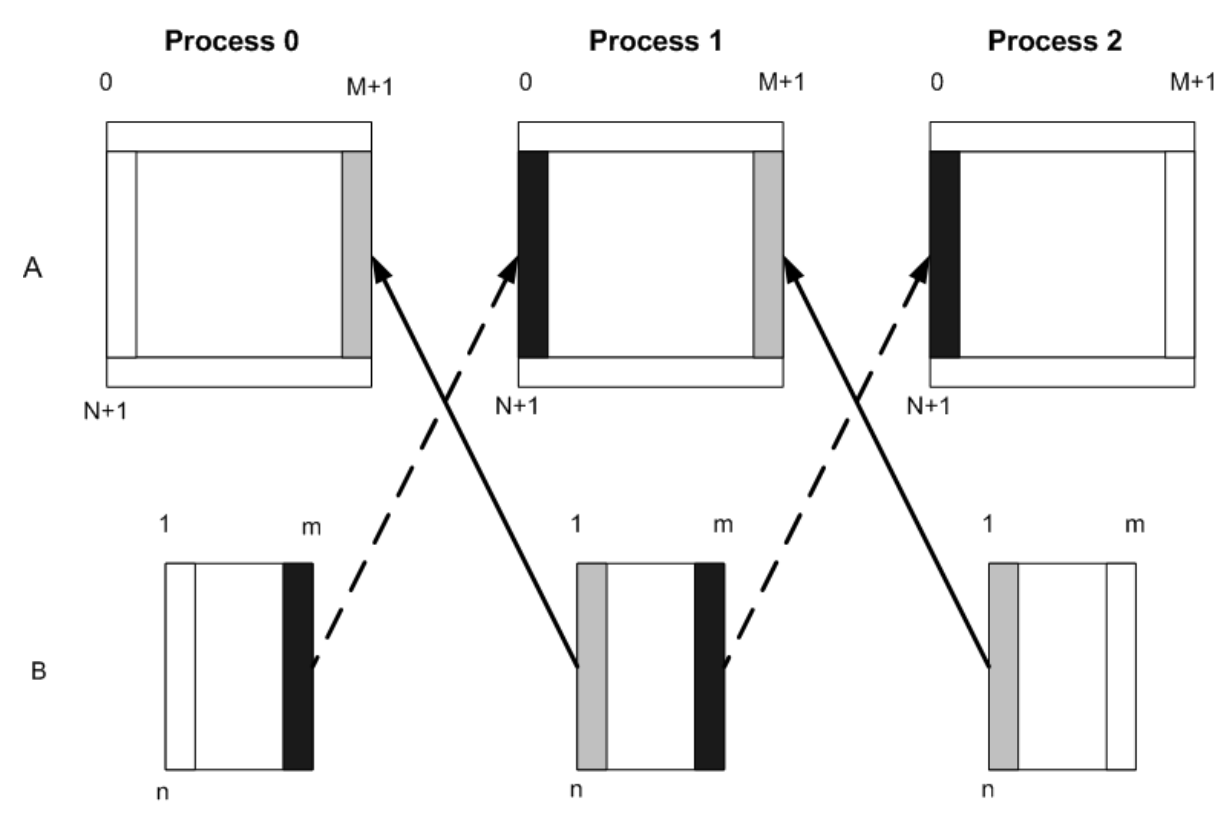

Fig. 1. Block partitioning with overlap and communication pattern

\section{Results}

A $50 \mathrm{~cm}$ long and $10 \mathrm{~cm}$ high channel is used for the detonation channel and the computational domain is discretized into $100 \times 150$ grid cells. The grids are clustered near the walls to capture the boundary layer. Adiabatic wall boundary conditions are imposed on the upper and lower sides of the computational domain whereas supersonic inflow and outflow were imposed on the left and right side respectively, with an ambient pressure of $1 \mathrm{~atm}$ imposed at the outlet of the channel. Pre-mixed hydrogen-air gas at an ambient pressure of $2 \mathrm{~atm}$ and temperature of $500 \mathrm{~K}$ is set to flow into the channel with a incoming Mach number of 2 . The mixture is ignited by a localized hot-spot at approximately $0.1 \mathrm{~m}$ into the channel from the left end. The hot spot is $1 \mathrm{~mm}$ long and $5 \mathrm{~mm}$ high with a pressure of $30 \mathrm{~atm}$ and a temperature of $3000 \mathrm{~K}$. Detonation waves are immediately generated and the initiation and propagation are shown in pressure contour plots in figure 2 .

The parallel algorithm was tested on the LoneStar cluster in the Texas Advanced Computing Center, a part of the national TeraGrid project, for benchmarking as well as scalability purposes. Figure 3 illustrated the scalability obtained from the parallel algorithm over a range of processors and increasing iterations. It is observed that with the present algorithm, maximum scalability was obtained when more iterations were computed, which indicates the good performance of the MPI parallel subroutines. As with any parallel algorithm, there would be a point where scalability reduces and the 

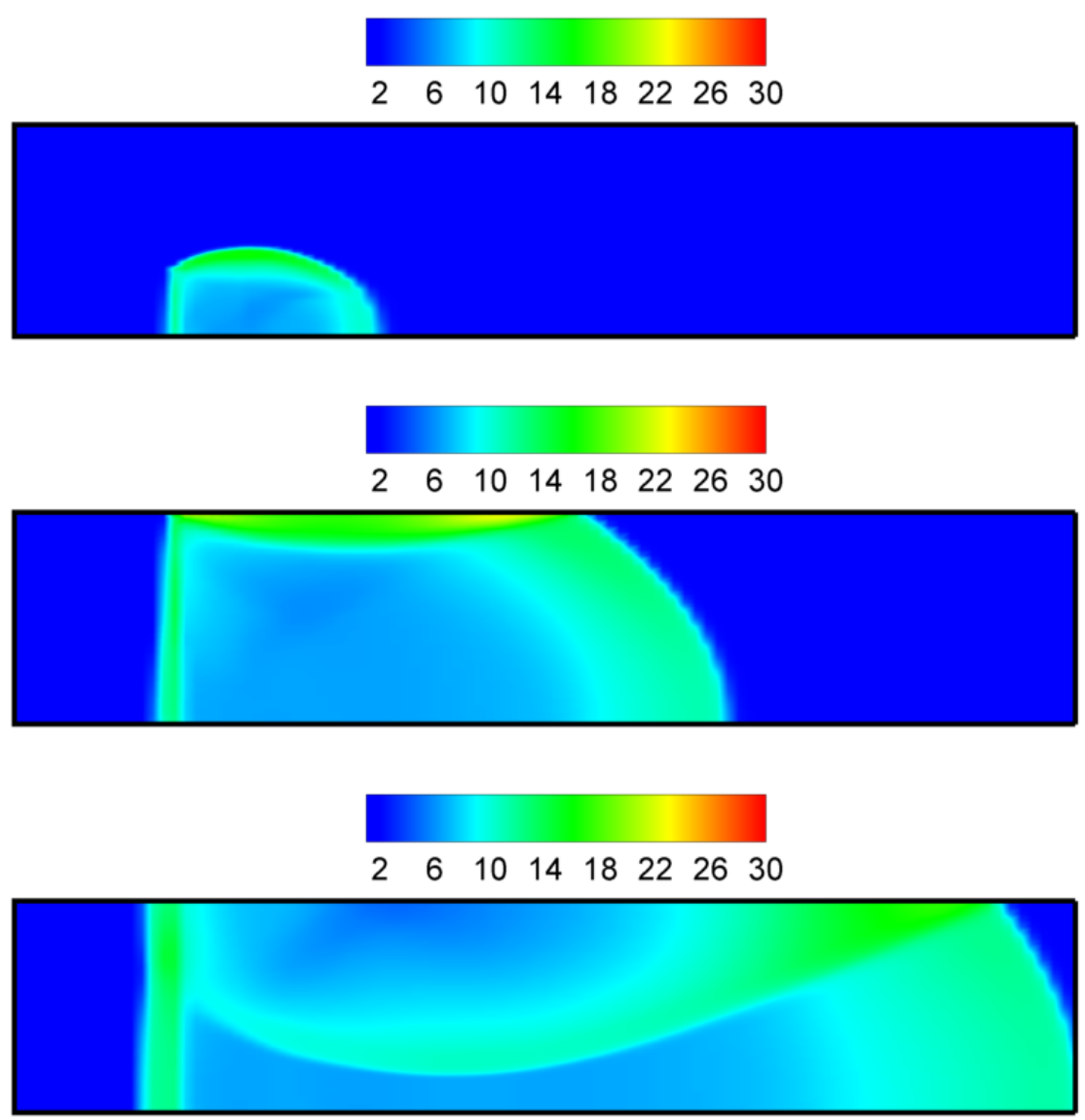

Fig. 2. Pressure contours for a detonation wave propagation in a channel

ensuing communications bottleneck would actually increase computation time. Figure 4 illustrates the issue of blockage and communication meltdown arising due to increasing communication messages with more number of processors. Figure 5 shows the memory usage of the process threads vary linearly with increasing processors indicating that the algorithm performs effective memory distribution.

The implemented algorithm has proven to be highly efficient and reliable in terms of accuracy and reduces computation time due to good scalability. A detailed study involving computation timelines has been performed and found out to be reasonable within bounds at evidenced by the results.

Acknowledgement. The authors acknowledge the support of the Texas Advanced Computing Center for the computations reported here.

\section{References}

1. Chapman, D.L.: On the rate of explosion in gases. In: Philos. Mag., 47, pp. 90-104 (1899) 


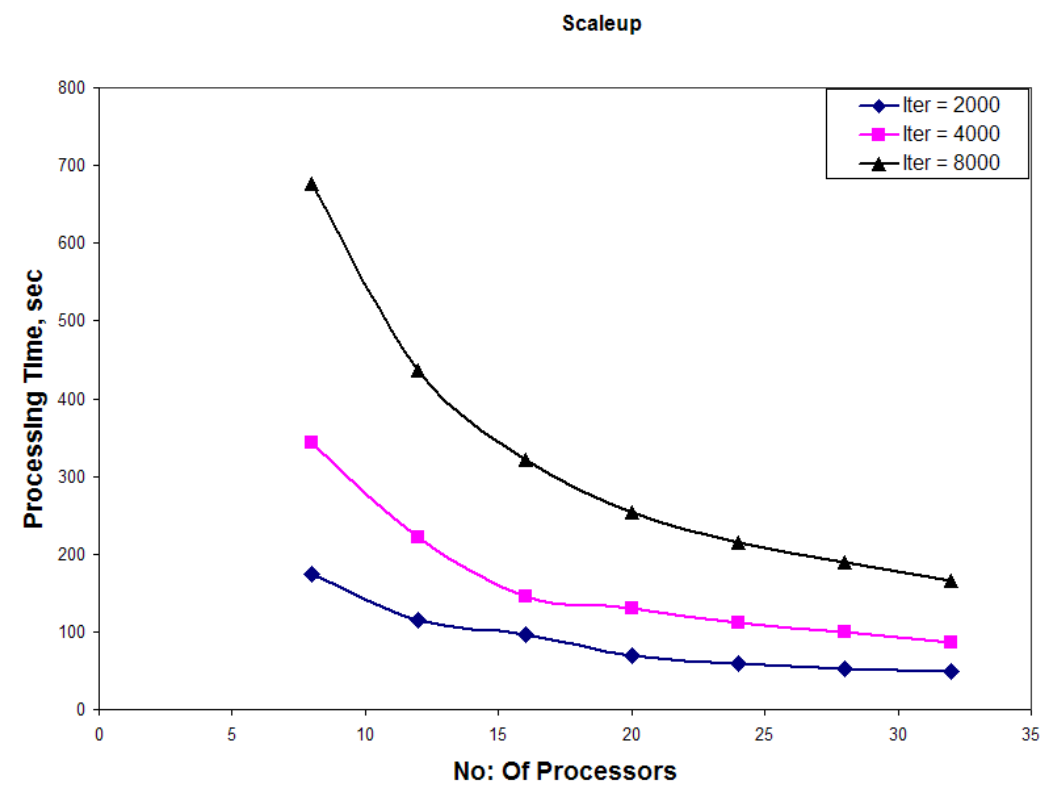

Fig. 3. Scaleup diagram for varying iterations

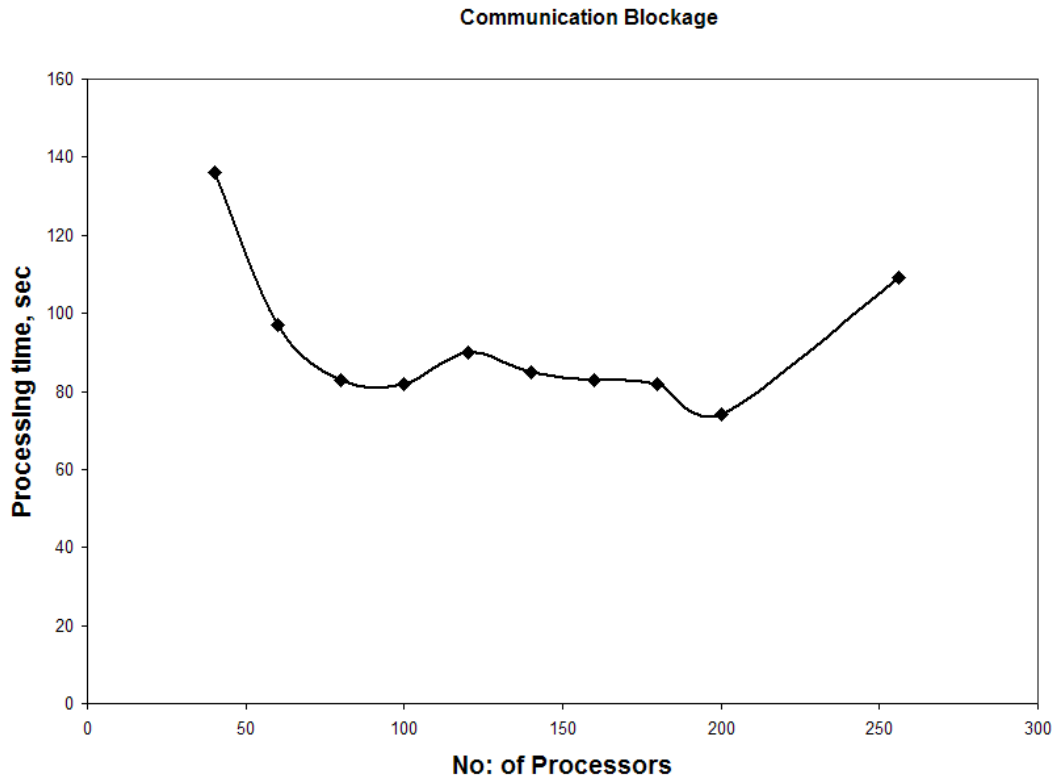

Fig. 4. Communication blockage

2. Jouguet E.: On the propagation of chemical reactions in gases. In: J. de Mathematiques Pures et Appliquees, 1, pp. 347-425 (1906)

3. Zel'dovich, Ya.B.: On the theory of the propagation of detonations on gaseous system. In: Zh. Eksp. Teor. Fiz., 10, pp. 542-568 (1940) 


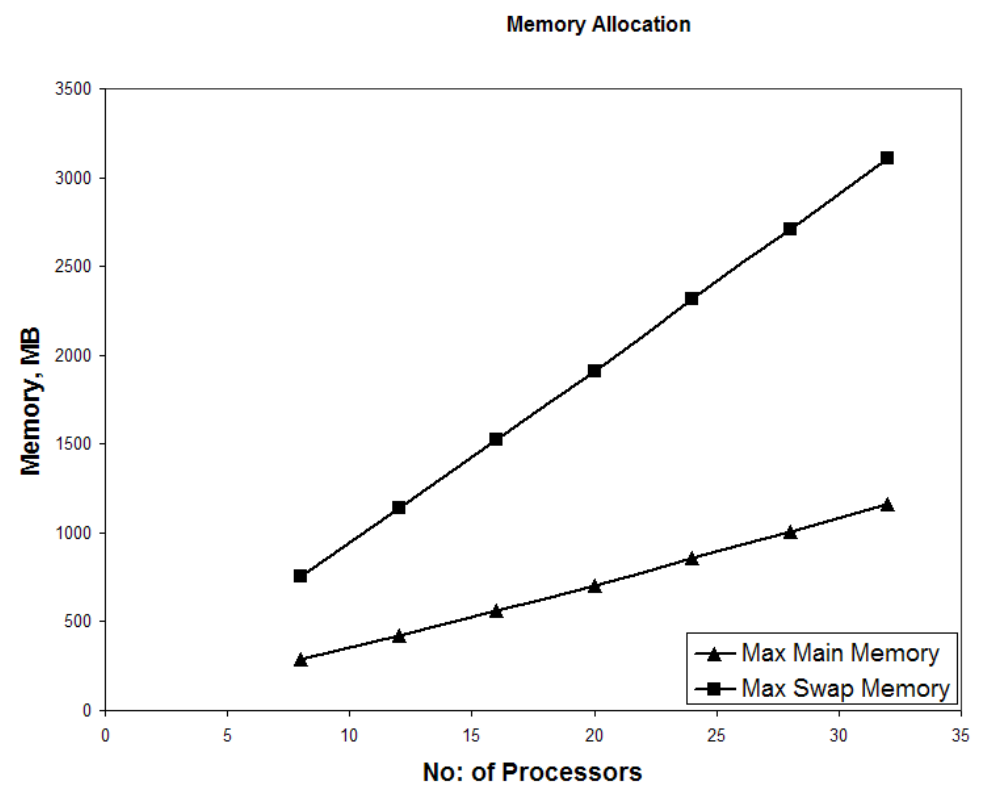

Fig. 5. Main memory and swap memory usage by the process threads

4. von Neumann, J.: Theory of detonation waves. Progress Report to the National Defense Research Committee Div. B, OSRD-549, (April 1, 1942. PB 31090); In: Taub, A.H. (ed). John von Neumann: Collected Works, 1903-1957, Vol. 6, Pergamon Press, New York, 1963

5. Döring, W.: On detonation processes in gases. In: Ann. Phys., 43, pp. 421-436 (1943)

6. Arnold G., Eicker N., Lippert T., Schilling K. (2001): Cluster computing vs. Cray T3E-a case study from numerical field theory In: Proc 9th Euromicro Workshop on Parallel and Distributed Processing, Mantova, Italy, pp. 475-479, IEEE Computer Soc, Los Alamitos, California, USA, (Feb 7-9 2001)

7. Ravindran, P.: Numerical simulation of pulse detonation phenomena in a parallel environment. MSAE thesis, University of Texas at Arlington, Arlington, Texas, USA (December 2005)

8. Gropp W., Lusk E., Doss N., Skjellum A.: A high-performance, portable implementation of the MPI message passing interface standard, Parallel Computing, 22(6), pp. 789-828 (1996)

9. Gropp W., Lusk E., Skjellum, A.: Using MPI, 2nd ed. (MIT Press, 1999)

10. Vincenti, W.G., Kruger, C.H.: Introduction to Physical Gas Dynamics (Krieger Publishing Company, 1965)

11. Bussing, T., Murman, E.: Finite volume method for the calculation of combustible chemically reacting flows AIAA J., 26(9), pp. 1070-1078 (1988)

12. Grossmann, B., Cinella, P.: Flux-split algorithms for flows with nonequilibrium chemistry and vibrational relaxation. In: J. Comp. Phys., 88(1), pp. 131-168 (1990) 